\title{
SINGLE CRYSTAL CVD DIAMOND DETECTORS FOR HADRON PHYSICS
}

\author{
MICHAL POMORSKI*, E. BERDERMANN, M. CIOBANU, B. E. FISHER, \\ A. MARTYMYIANOV, P. MORITZ, M. REBISZ, B. VOSS \\ Gesellschaft fur Schwerionenforschung (GSI), Planckstr. 1 \\ Darmstadt, 64291,Germany \\ J. MORSE, M. SALOME \\ European Synchrotron Facility (ESRF) \\ Grenoble, 38043 CEDEX, 6 rue Jules Horowitz, France
}

\begin{abstract}
We report on characterization of several single crystals CVD diamond detectors tested for the use in heavy-ion spectrometry and minimum ionizing particles time of flight measurements. An energy resolution of $16 \mathrm{keV}$ (FWHM) is achieved using a ${ }^{241} \mathrm{Am} \alpha$ source, which is comparable to the energy resolution of silicon detectors. The collected charge maps, measured using ${ }^{12} \mathrm{C}$ micro beams confirm the excellent homogeneity of the novel material. Using low impedance broadband electronics and a transient-current technique the mobility and saturation drift velocity of electrons and holes are estimated.
\end{abstract}

\section{Introduction}

Due to its remarkable physical properties [1-3] diamond is one of the most promising wide band-gap materials for particle detection in present and future hadron physics experiments, where radiation hardness and speed are the crucial requirements. Recent progress in the growth of ultra pure Single-Crystal CVD diamond [4] has opened perspectives to fast and high-resolution radiation sensors based on this novel type of artificial diamond

\section{Detector Preparation and Experimental setup}

The samples characterized in this work are single crystal diamond films made by Chemical Vapor Deposition (CVD) at Element Six, Ascot, UK. Cleaning and oxidation in boiling mixture of $\mathrm{H}_{2} \mathrm{SO}_{4} / \mathrm{KNO}_{3}$ is performed before electrodes in "sandwich" configuration are applied by sputtering. Different metallic layers have been tested consisting of: $\operatorname{Ti}(20 \mathrm{~nm}): \operatorname{Pt}(30 \mathrm{~nm}): \mathrm{Au}(100 \mathrm{~nm})$, $\mathrm{Cr}(50 \mathrm{~nm}): \mathrm{Au}(100 \mathrm{~nm})$ and $\mathrm{Al}(100 \mathrm{~nm})$. No differences in IV characteristics or 
other detector properties are found so far for various metallization.
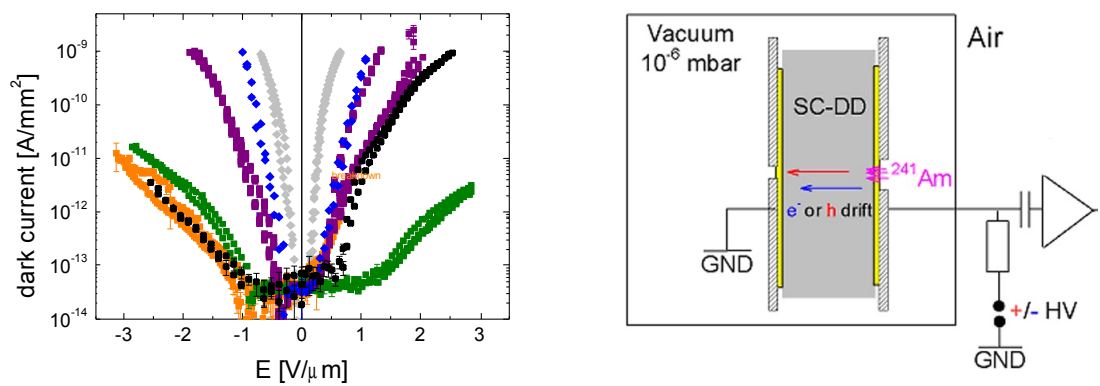

Fig. 1. (left) IE characteristics for several SC diamond samples. Unexpected huge spread between samples and low break down field is found for most of the tested samples; (right) Mounting of the samples and geometry of irradiation.

Before detector irradiation the I-V characteristics is probed in order to determine the safe range of detector HV bias. The current-voltage curves of various samples, normalized to the contact surface are shown in Fig. 1 (left). For most of the samples, unexpected huge spread and low break-down fields have been observed. Optical microscopy and AFM studies (not presented here) of the samples surfaces show damaged regions probably due to polishing processes after diamond growth. Such undefined state of the interlayer diamond-metal can be the reason for the observed behavior. Further work is ongoing indenting to improve post-growth surface preparation and thus the electrode metallization.

The diamond detector is clamped between two printed circuit boards (Fig.1, right). This mounting provides electrical contact and $\alpha$ particles collimation as well. A ${ }^{241} \mathrm{Am} \alpha$-source is placed above the collecting electrode. Impinging $\alpha$ particles of $5.468 \mathrm{MeV}$ have a short range of $\sim 12 \mu \mathrm{m}$ in diamond, thus, for the presented irradiation geometry, the choice of $\mathrm{HV}$ polarity enables mainly holes drift at positive bias, and electrons drift at negative potential [5].

\section{Charge Collection Efficiency (CCE) and Energy resolution}

Using a classical nuclear electronics chain, cross-calibrated by silicon detector and spectroscopic pulser [6], the collected-charge characteristics is measured. Results for 4 different samples are displayed in Fig. 2 (left). Blue curves correspond to the electrons collection efficiency and red data to the efficiency of holes. In all cases we observe saturation of collected charge to $\sim 68.7 \mathrm{fC}$ at relatively low bias. 

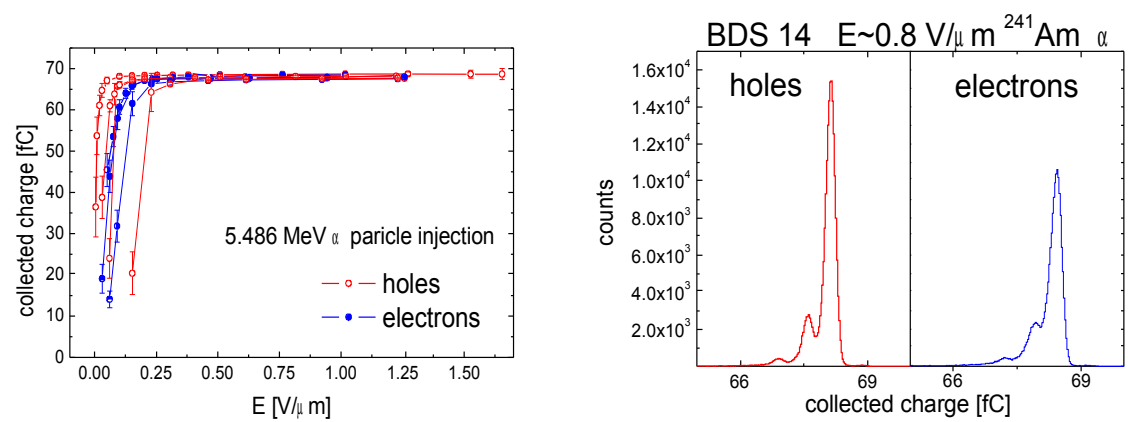

Fig. 2 (left) Collected charge characteristics of electrons (blue), and holes (red) obtained from four SC diamond detectors. (right) The $\alpha$-spectra recorded for holes and electrons drift, respectively. All satellite lines are distinguished. The energy resolution (FWHM) for holes drifts is $\Delta \mathrm{E} / \mathrm{E} \sim 0.003$.

Good energy resolution of monoenergetic $\alpha$-particles impinging randomly an area of a few square millimeters, is an indicator of the diamond bulk's homogeneity. The spectrum presented in Fig. 2 (left) is an evidence for excellent homogeneity of this diamond crystal, as well as for long term stability (time of measurements $>12 \mathrm{~h}$ ). An energy resolution of $\Delta \mathrm{E}_{\mathrm{D}}(\mathrm{FWHM})=17 \mathrm{keV}$ is extracted from a Gaussian fit of the ${ }^{241} \mathrm{Am}$ main peak, which is comparable to the value of $\Delta \mathrm{E}_{\mathrm{SiD}}=14 \mathrm{keV}$ obtained with a silicon detector under same experimental conditions. In addition having such excellent energy resolution and detection stability we can assume charge collection efficiency close to $100 \%$ at plateau of curves presented in Fig.2 (left). Thus estimation of average energy needed for e-h pair creation is possible, which amounts to $\sim 12.8 \mathrm{eV} / \mathrm{e}-\mathrm{h}$.

\section{Timing Properties - Time of Flight Technique}

We are using the intrinsic ToF technique [6-8], in order to probe the timing properties of SC diamond detectors. The electric field is applied along the $\langle 100>$ crystal direction. The average (over 500 single shots) current signals, recorded with a DBA-II broad band voltage amplifier of $50 \Omega$ impedance [7] and a digital scope of $3 \mathrm{GHz}$ bandwidth, are presented in Fig. 3 for holes- (red) and electron drift (blue). The FWHM of these signals marks the transition time $t_{t r}$ of the charge carriers cloud between the moment of its generation near one contact and its arrival to the opposite electrode. The flat top of the signals indicate a uniform internal electric field (no space charge and negligible trapping). Thus the drift velocity $v_{\mathrm{dr}}$ of carriers is constant at fixed bias and can be easily calculated from the simple relation $v_{\mathrm{dr}}=\mathrm{d} / \mathrm{t}_{\mathrm{tr}}$, where $\mathrm{d}$ is the sample thickness. The dependence of the drift velocity from the applied external electric field $\mathrm{E}$ for six tested samples is displayed in Fig. 3 (right). 

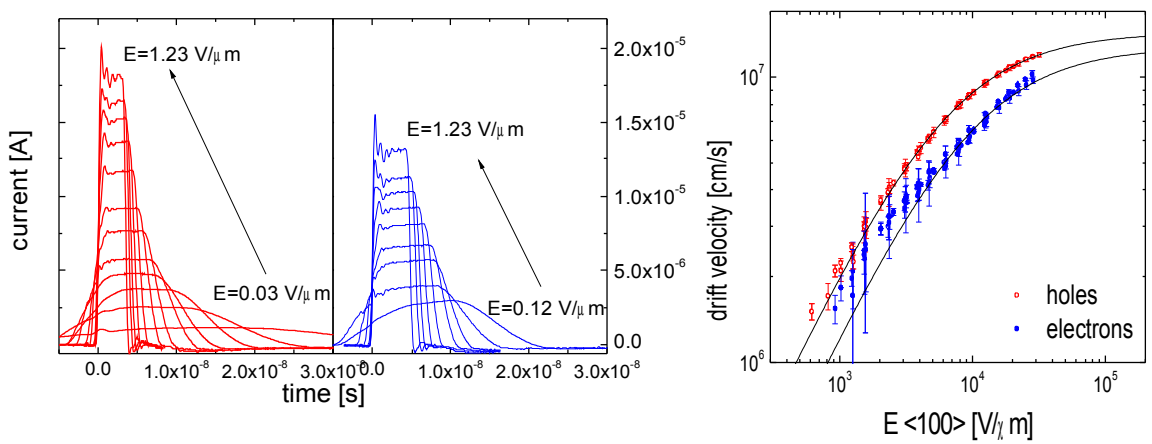

Fig.3 (left) An example of broad-band current signals (average from 500 single shots) for holes- (red) and electrons (blue) drift at increasing electric field. The oscillation on top is due to a weak $50 \Omega$ mismatching. (right) Charge carriers drift velocity as a function of electric field for 6 tested SC diamond samples.

Since the transport process at very high electric field is difficult to be theoretically described, the data are fitted by the empirical equation [6-10]:

$$
v_{d r}(E)=\frac{\mu_{0} E}{1+\frac{v_{s}}{\mu_{0}} E}
$$

Where $\mu_{0}$ - low field mobility, $v_{\text {sat }}$-saturation velocity.

Excelent reproducibility of the drift velocity of holes is found for various samples, in contrast to the electrons, where spread of experimental points affects significantly the precision of the fit. Thus, the extrapolated parameters are given for an interval. Table 1 shows the timing parameters, extracted for several tested samples.

Table1. Measured efective mobilities $\left(\boldsymbol{\mu}_{\mathrm{eff}}\right)$ and drift velocities $\left(\boldsymbol{v}_{\mathrm{dr}}\right)$ at typical detector operation range $0.6<\mathrm{E}<2[\mathrm{~V} / \mu \mathrm{m}]$, as well as low filed mobilities $\left(\boldsymbol{\mu}_{\mathrm{o}}\right)$ and saturation velocities $\left(\boldsymbol{v}_{\mathrm{sat}}\right)$ extrapolated from fits of the experimental data.

\begin{tabular}{|r|c|c|c|c|}
\hline & \multicolumn{2}{|c|}{ Measured } & \multicolumn{2}{c|}{ Extrapolated } \\
\cline { 2 - 5 } & $\boldsymbol{\mu}_{\text {eff }}\left[\mathbf{c m}^{2} / \mathbf{V s}\right]$ & $\boldsymbol{v}_{\mathrm{dr}}[\boldsymbol{\mu m} / \mathbf{n s}]$ & $\boldsymbol{\mu}_{\mathbf{0}}\left[\mathbf{c m}^{2} / \mathbf{V s}\right]$ & $\boldsymbol{V}_{\text {sat }}[\mathbf{c m} / \mathbf{s}]$ \\
\hline electrons & $1000-450$ & $50-90$ & $\sim 1300-3000$ & $1.3-1.9^{*} 10^{7}$ \\
\hline holes & $1400-550$ & $70-110$ & $\sim 2400$ & $\sim 1.4^{*} 10^{7}$ \\
\hline
\end{tabular}

\section{Mapping with heavy-ion micro beams}

In order to study spatial homogeneity, a SC CVD diamond detector has 
been irradiated with ${ }^{12} \mathrm{C}^{2+}$ ions of a kinetic energy $\mathrm{E}=9.5 \mathrm{~A} \mathrm{MeV}$ at the micro beam facility of GSI. The irradiated area was $60 \times 93\left[\mu \mathrm{m}^{2}\right]$ and the sample was scanned in a $1 \mu \mathrm{m}$ raster. Classical charge sensitive nuclear electronics was used for the signal readout. The obtained 3D distribution of the detector's pulse heights is presented in Fig. 4 (left); no regions of lower ( $\pm 2 \%)$ CCE in scanned area are observed.

In Fig. 4 (right) the calibrated 1D spectrum is shown, where the left peak is the pulser line used to control the stability of the electronics, the intermediate one the ${ }^{12} \mathrm{C}^{2+}$ ion distribution and the right one due to an unavoidable beam contamination $(\sim 0.6 \%)$ with ${ }^{18} \mathrm{O}^{+3}$ ions having the same $\mathrm{A} / \mathrm{Q}$. The unexpected oxygen peak has been used for energy calibration of the spectrum. The obtained energy resolution of the ${ }^{12} \mathrm{C}$ peak is $\Delta \mathrm{E} / \mathrm{E}=0.01$.
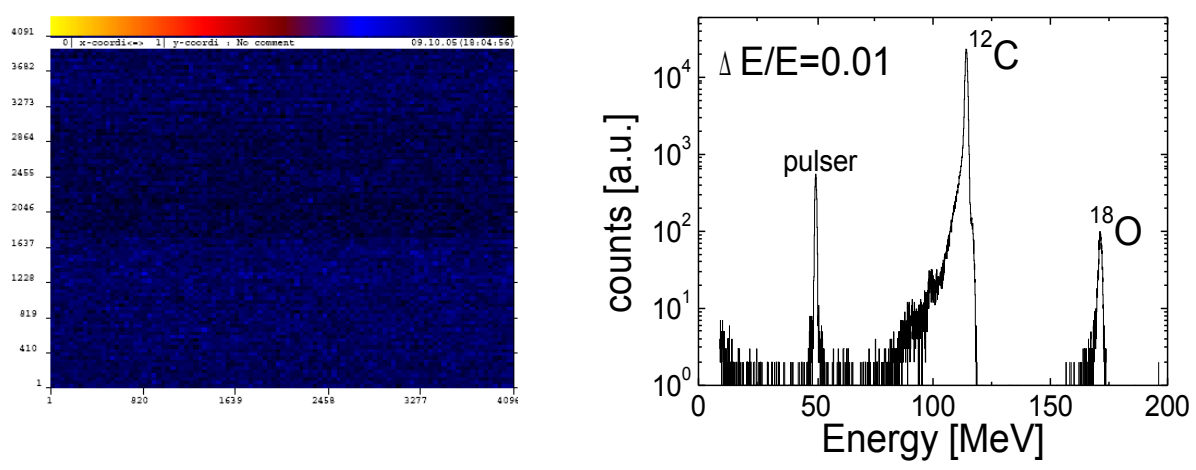

Fig.4 (left) Pulse height map of a SC CVD diamond detector measured with ${ }^{12} \mathrm{C}$ ions of $\mathrm{E}=9.5 \mathrm{AMeV}$ using nuclear spectroscopy read-out. The irradiated area was $60 \times 93\left[\mu \mathrm{m}^{2}\right]$. (right) The Corresponding calibrated $1 \mathrm{D}$ spectrum.

The obtained map and the good energy resolution of the ${ }^{12} \mathrm{C}$ peak confirm the excellent homogeneity of single crystal diamond, which was already indicated during the irradiation tests with $\alpha$-particles.

\section{Summary and Outlook}

The study conducted here gives a clear evidence that SC CVD diamond is a competitive material for silicon pin diodes, frequently used in hadron physics experiments. Most of the tested samples show stable operation, negligible charge carriers trapping and material homogeneity. The energy resolution found for alpha particles of $\mathrm{E}_{\alpha} \approx 5.5 \mathrm{MeV}$ and ${ }^{12} \mathrm{C}$ ions of $\mathrm{E}=9.5 \mathrm{AMeV}$ is close to the values obtained from silicon detectors. The timing properties of 
diamond are superior to silicon. Fast and comparable drift velocity for holes and electrons is measured, which results in the absence of slow signal components. However, the low e-h pair creation compared to silicon, is a drawback for MIP- and weakly-ionizing particles detection.

The important radiation hardness tests are planned for the next future. Nevertheless, considering the extreme physical properties of diamond and the experimental data obtained from polycrystalline CVD diamond films [12], a sufficient high radiation dose tolerance is expected.

\section{Acknowledgments}

This work is supported by the EC Integrated Infrastructure Initiative Hadron Physics Project under contract number: RII 3-CT-2004-506078.The authors express their gratitude to Element Six, Ascot, UK for the SC diamond samples provision and to the Target Laboratory crew of GSI for the samples metallization.

\section{References}

1. E. Berdermann, et al., ICATPP,2001, p246-251.

2. M. Marinelli,et al, Diamond Related Mater. 7 (1998) 519.

3. J. Isberg et al., Science 297 (2002).

4. Element Six, Ascot, UK

5. S. Ramo, Proc. IRE 27, 584 (1939).

6. M. Pomorski, E. Berdermann phys. stat. sol. (a) 202, No. 11, 2199-2205 (2005)

7. P. Moritz, E. Berdermann, K. Blasche, H. Stelzer, and B. Voss, Diam. Relat. Mater. 10, 1765 (2001).

8. H. Pernegger, et al, Journal of Applied Physics 97, 073704 (2005)

9. C. Jacoboni, C. Canali, G. Ottaviani, and A. Alberigi Quaranta, Solid-State Electron. 20, 77 (1977).

10. T. Lari, A. Oh, N. Wermes, H. Kagan, M. Keil, and W. Trischuk, Nucl. Instrum. Methods A 537, 581 (2005)

11. E. Berdermann, K. Blasche, P. Moritz, H. Stelzer1, and B. Voss, Diam. Relat. Mater. 10, 1770 (2001).

12. W. Adam et al. NIM A: , Vol. 476, Issue 3, 11 January 2002, Pages 686-693 